\title{
Häufig benutzte Formeln der Vektorrechnung
}

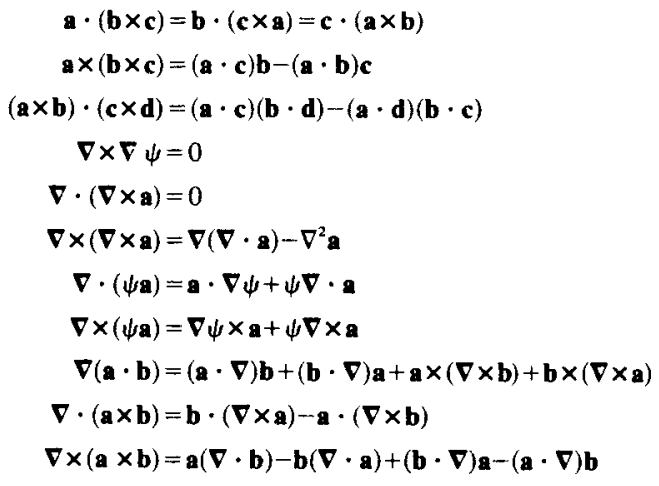

Ist $\mathbf{x}$ der Ortsvektor eines Punktes bezüglich eines bestimmten Koordinatenursprungs, ferner $\mathbf{r}=|\mathbf{x}|$ der Betrag dieses Vektors und $\mathbf{n}=\mathbf{x} / \mathbf{r}$ ein Einheitsvektor in Richtung von $\mathbf{x}$, dann gilt:

$$
\begin{array}{cc}
\nabla \cdot \mathbf{x}=3 & \boldsymbol{\nabla} \times \mathbf{x}=0 \\
\boldsymbol{\nabla} \cdot \mathbf{n}=\frac{2}{r} & \boldsymbol{\nabla} \times \mathbf{n}=0 \\
(\mathbf{a} \cdot \boldsymbol{\nabla}) \mathbf{n}=\frac{1}{r}[\mathbf{a}-\mathbf{n}(\mathbf{a} \cdot \mathbf{n})] \equiv \frac{\mathbf{a}_{-}}{r}
\end{array}
$$

